\title{
PRODUCTS OF IDEMPOTENTS IN CERTAIN SEMIGROUPS OF TRANSFORMATIONS
}

\author{
by J. M. HOWIE \\ (Received 13th February 1970)
}

In an earlier paper (5) a description was given in set-theoretic terms of the semigroup generated by the idempotents of a full transformation semigroup $\mathscr{T}_{X}$, one of the results being that if $X$ is finite then every element of $\mathscr{T}_{X}$ that is not bijective is expressible as a product of idempotents. In view of this it was natural to ask whether by analogy every singular square matrix is expressible as a product of idempotent matrices. This is indeed the case, as was shown by J. A. Erdos (2). Magill (6) has considered products of idempotents in the semigroup of all continuous self-maps of a topological space $X$, but a comparable characterization of products of idempotents in this case appears to be extremely difficult, and no solution is available yet.

In this paper I consider the semigroup $\mathcal{O}_{X}$ of order-preserving mappings of a totally ordered set $X$. In the finite case, where we may assume that $\mathcal{O}_{X}$ is the set of order-preserving mappings of $\{1,2, \ldots, n\}$, the answer to our question is easy: every element of $\mathcal{O}_{x}$ is expressible as a product of idempotents in $\mathcal{O}_{x}$. It is also possible to obtain formulae (in terms of $|X|$ ) for the number of elements in $\mathcal{O}_{X}$ and the number of idempotents in $\mathcal{O}_{X}$.

The most natural case to consider next is where $X$ has order-type $\omega$, but here the situation is much more difficult. The result in $\S 3$ refers not to $\mathscr{O}_{X}$ itself but to a subsemigroup $\mathscr{B}_{X}$ consisting of increasing mappings $\alpha$ for which the sets $y \alpha^{-1}(y \in X \alpha)$ are bounded in size.

\section{Finite sets}

The principal result of this section is

Theorem 1.1. If $X$ is a finite totally ordered set, then every element of the semigroup $\mathcal{O}_{X}$ of order-preserving mappings of $X$ into itself is expressible as a product of idempotents in $\mathcal{O}_{X}$.

Let us assume throughout that $X=\{1,2, \ldots, n\}$.

As in (5), we define $Z(\alpha)$, for $\alpha$ in $\mathcal{O}_{X}$, to be $X \backslash X \alpha$, and refer to $|Z(\alpha)|$ as the defect of $\alpha$. The result follows from Lemmas 1.2 and 1.3 below, since the unique element of defect zero in $\mathcal{O}_{X}$ is an idempotent. The lemmas in fact imply the stronger result that every element of $\mathcal{O}_{X}$ other than the identical mapping is expressible as a product of idempotents of defect 1 . 
Lemma 1.2. An element of $\mathcal{O}_{X}$ of defect greater than 1 is expressible as a product of idempotents of defect 1 and a single element of defect 1 .

Lemma 1.3. An element of $\mathcal{O}_{X}$ of defect 1 is expressible as a product of idempotents of defect 1 .

The proofs of these lemmas are inductive in nature. First, it is clear that Lemma 1.2 will follow if we establish the following rather more technical result.

Lemma 1.4. If $\alpha \in \mathcal{O}_{X}$ has defect $k>1$ then there exist an integer $r \geqq 1$ and idempotents $\varepsilon_{1}, \varepsilon_{2}, \ldots, \varepsilon_{r} \in \mathcal{O}_{X}$ of defect 1 such that

where $\beta$ has defect $k-1$.

$$
\alpha=\varepsilon_{1} \beta \varepsilon_{2} \ldots \varepsilon_{r}
$$

To prove this, consider an element $\alpha$ of defect $k(>1)$ and let

$$
P(\alpha)=\left\{y \in X \alpha:\left|y \alpha^{-1}\right| \geqq 2\right\} ;
$$

$P(\alpha) \neq \varnothing$ since $\alpha$ is not one-one. Let

$$
d_{\alpha}=\min \{|z-p|: z \in Z(\alpha), p \in P(\alpha)\} ;
$$

$d_{\alpha} \geqq 1$ since $Z(\alpha) \cap P(\alpha)=\varnothing$. We shall prove Lemma 1.4 by induction on $d_{\alpha}$.

First, if $d_{\alpha}=1$ we can assume without essential loss of generality that there exists $p$ in $P(\alpha)$ for which $p+1 \in Z(\alpha)$. [The other case, where $p-1 \in Z(\alpha)$, can be treated analogously.] Let $q=\max \{x \in X: x \alpha=p\}$. Since $p \alpha^{-1}$ contains more than one element, there exists $q^{\prime} \leqq q-1$ such that $q^{\prime} \alpha=p$. Hence

$$
p=q^{\prime} \alpha \leqq(q-1) \alpha \leqq q \alpha=p,
$$

and so $(q-1) \alpha=p$. The mapping $\varepsilon: X \rightarrow X$ defined by

$$
q \varepsilon=q-1, \quad x \varepsilon=x \quad(x \neq q)
$$

is an idempotent of defect 1 in $\mathcal{O}_{X}$. If we define $\beta: X \rightarrow X$ by

$$
q \beta=p+1, \quad x \beta=x \alpha \quad(x \neq q),
$$

then $\beta \in \mathcal{O}_{X},|Z(\beta)|=|Z(\alpha)|-1=k-1$, and $\alpha=\varepsilon \beta$.

If $d_{\alpha}>1$ we can suppose without essential loss of generality that there exists $z \in Z(\alpha)$ such that

$$
z+1, \ldots, z+d_{\alpha}-1 \in X \alpha \backslash P(\alpha), \quad z+d_{\alpha} \in P(\alpha) .
$$

Then $(z+1) \alpha^{-1}$ consists of a single element $t$ (say), and $(t+1) \alpha=z+2$. If $\gamma: X \rightarrow X$ is defined by

$$
t \gamma=z, \quad x \gamma=x \alpha \quad(x \neq t),
$$

then $\gamma \in \mathcal{O}_{X}$ and $|Z(\gamma)|=|Z(\alpha)|=k$. Also $z+1 \in Z(\gamma), z+d_{\alpha} \in P(\gamma)$ and so $d_{\gamma}=d_{\alpha}-1$. If $\eta: X \rightarrow X$ is defined by

$$
z \eta=z+1, \quad x \eta=x \quad(x \neq z),
$$

then $\eta$ is an idempotent of defect 1 in $\mathcal{O}_{X}$, and $\gamma \eta=\alpha$. 
If we suppose inductively that there exist idempotents $\varepsilon_{1}, \varepsilon_{2}, \ldots, \varepsilon_{s}$ of defect 1 in $\mathcal{O}_{X}$ such that $\gamma=\varepsilon_{1} \beta \varepsilon_{2} \ldots \varepsilon_{s}$, where $\beta$ has defect $k-1$, it now follows that

$$
\alpha=\varepsilon_{1} \beta \varepsilon_{2} \ldots \varepsilon_{s} \eta \text {. }
$$

This completes the proof of Lemma 1.4 and thus Lemma 1.2 is established.

To prove Lemma 1.3, let us first define (as in (5)) the shift $s_{\alpha}$ of an element $\alpha$ in $\mathcal{O}_{X}$ to be the cardinal of the set $S(\alpha)=\{x \in X: x \alpha \neq x\}$. If $s_{\alpha}=1$ then $\alpha$ is an idempotent of defect 1. To see this, let us write $S(\alpha)=\{u\}$. If $x \neq u$ then $x \alpha=x \alpha^{2}=x$; on the other hand $u \alpha \neq u$ and so $u \alpha^{2}=u \alpha$. Thus $\alpha$ is idempotent, and, since the range of $\alpha$ includes every element of $X$ except $u$, the defect must be 1 .

In view of this, Lemma 1.3 will follow if we establish

Lemma 1.5. If $\alpha\left(\in \mathcal{O}_{X}\right)$ has defect 1 and if $s_{\alpha}>1$ then there exists an idempotent $\varepsilon$ of defect 1 in $\mathcal{O}_{X}$ and an element $\beta$ of defect 1 in $\mathcal{O}_{X}$ such that $\alpha=\varepsilon \beta$ and

$$
s_{\beta}=s_{\alpha}-1 \text {. }
$$

Proof. If $\alpha$ has defect 1 then there is a unique $u$ in $X$ such that $u \alpha=(u+1) \alpha$. (The two elements of $X$ with the same image must be adjacent since $\alpha$ is orderpreserving.) Suppose that $Z(\alpha)=\{v\}$. If $u<v$, then

if $u \geqq v$, then

$$
\begin{array}{ll}
x \alpha=x & (x \leqq u) \\
x \alpha=x-1 & (u+1 \leqq x \leqq v), \\
x \alpha=x & (x \geqq v+1)
\end{array}
$$

$$
\begin{array}{ll}
x \alpha=x & (x<v), \\
x \alpha=x+1 & (v \leqq x \leqq u), \\
x \alpha=x & (x \geqq u+1) .
\end{array}
$$

If $u<v$ we define $\varepsilon: X \rightarrow X$ and $\beta: X \rightarrow X$ by

$$
\begin{array}{lll}
(u+1) \varepsilon=u, & x \varepsilon=x & (x \neq u+1), \\
(u+1) \beta=u+1, & x \beta=x \alpha & (x \neq u+1) ;
\end{array}
$$

then $\varepsilon$ and $\beta$ are both in $\mathcal{O}_{X}$ and of defect $1, \varepsilon$ is an idempotent, $s_{\beta}=s_{\alpha}-1$, and $\alpha=\varepsilon \beta$.

If $u \geqq v$ then the desired result is obtained if we define $\varepsilon: X \rightarrow X$ and $\beta: X \rightarrow X$ by

$$
\begin{array}{ll}
u \varepsilon=u+1, & x \varepsilon=x \quad(x \neq u), \\
u \beta=u, & x \beta=x \alpha \quad(x \neq u) .
\end{array}
$$

The proof of Theorem 1.1 is now complete.

\section{Combinatorial results}

It is well-known (and indeed obvious) that the full transformation semigroup on a set $X$ of $n$ elements has order $n^{n}$. The number of idempotents in $\mathscr{T}_{X}$ is not

$$
\text { E.M.S. }-\mathbf{P}
$$


so obvious, and has been investigated by Harris (3), Harris and Schoenfeld (4) and Tainiter (8).

The first result of this section is

Theorem 2.1. If $|X|=n$ then $\left|\mathcal{O}_{X}\right|=\left(\begin{array}{c}2 n-1 \\ n-1\end{array}\right)$.

Proof. As before, we may assume that $X=\{1,2, \ldots, n\}$. If $\alpha \in \mathcal{O}_{X}$ then

$$
1 \leqq 1 \alpha \leqq 2 \alpha \leqq \ldots \leqq n \alpha \leqq n .
$$

For $r=1, \ldots, n-1$, define $g_{r}(\alpha)$, the gap of $\alpha$ at $r$, by

$$
g_{r}(\alpha)=(r+1) \alpha-r \alpha
$$

also, let $g_{0}(\alpha)=1 \alpha-1, g_{n}(\alpha)=n-n \alpha$. Clearly

$$
\sum_{r=0}^{n} g_{r}(\alpha)=n-1
$$

Notice that $\alpha$ is completely specified by the $(n+1)$-tuple $\left(g_{0}(\alpha), \ldots, g_{n}(\alpha)\right)$ of non-negative integers, and that any $(n+1)$-tuple of non-negative integers satisfying condition (2.2) defines an $\alpha$ in $\mathcal{O}_{X}$.

Now, the number of ways of arranging $n$ noughts and $n-1$ crosses is

$$
\frac{(2 n-1) !}{n !(n-1) !}=\left(\begin{array}{c}
2 n-1 \\
n-1
\end{array}\right)
$$

Each such arrangement is uniquely associated with an $\alpha$ in $\mathcal{O}_{X}$ if we take $g_{0}(\alpha)$ as the number of initial crosses, $g_{r}(\alpha)(r=1, \ldots, n-1)$ as the number of crosses between the $r$ th and the $(r+1)$ th nought, and $g_{n}(\alpha)$ as the number of final crosses. The result of the theorem follows.

Remark. Dr. J. Hunter has suggested an elegant alternative approach to the last part of this argument, based on the observation that

$$
\left|\left\{\left(x_{0}, \ldots, x_{n}\right): x_{r} \geqq 0, \sum_{r=0}^{n} x_{r}=n-1\right\}\right|
$$

is the coefficient of $x^{n-1}$ in $(1-x)^{-(n+1)}$.

Theorem 2.3. If $|X|=n$ then the number of idempotents in $\mathcal{O}_{X}$ is

$$
\phi(n)=\frac{1}{2^{n} \sqrt{5}}\left\{(3+\sqrt{5})^{n}-(3-\sqrt{5})^{n}\right\}=\frac{1}{2^{n-1}} \sum_{r=0}^{[1(n-1)]}\left(\begin{array}{c}
n \\
2 r+1
\end{array}\right) 3^{n-2 r-1} 5^{r}
$$

Proof. The first step is to write

$$
\phi(n)=\phi_{1}(n)+\phi_{2}(n)+\ldots+\phi_{n}(n),
$$

where $\phi_{r}(n)$ is the number of idempotents $\varepsilon$ in $\mathcal{O}_{X}$ for which $1 \varepsilon=r$. If $1 \varepsilon=r$ then $r \varepsilon=r$ and so in fact

$$
1 \varepsilon=2 \varepsilon=\ldots=r \varepsilon=r
$$




\section{PRODUCTS OF IDEMPOTENTS}

If we denote by $A_{r}$ the set of idempotents in $\mathcal{O}_{X}$ for which $1 \varepsilon=r$ (so that $\left|A_{r}\right|=\phi_{r}(n)$ ), we can express $A_{r}$ as a disjoint union

$$
A_{r}=A_{r, r} \cup A_{r, r+1} \cup \ldots \cup A_{r, n},
$$

where $A_{r, r+k}(k=0, \ldots, n-r-1)$ is the set of idempotents in $\mathcal{O}_{X}$ for which

$$
1 \varepsilon=\ldots=r \varepsilon=\ldots=(r+k) \varepsilon=r, \quad(r+k+1) \varepsilon>r,
$$

and where $A_{r, n}$ comprises the single element $\eta$ for which

$$
1 \eta=\ldots=n \eta=r .
$$
that

An element $\varepsilon$ in $A_{r, r+k}(k \neq n-r)$ does in fact possess the stronger property

$$
(r+k+1) \varepsilon \geqq r+k+1,
$$

for none of the elements $r+1, \ldots, r+k$ are mapped identically by $\varepsilon$ and so they cannot belong to its range. It follows that $\varepsilon$ maps the set $\{r+k+1, \ldots, n\}$ in an order-preserving fashion into itself. Moreover, a mapping

$$
\alpha=\left(\begin{array}{ccccccc}
1 & 2 & \ldots & r+k & r+k+1 & \ldots & n \\
r & r & \ldots & r & (r+k+1) \alpha & \ldots & n \alpha
\end{array}\right)
$$

in which $\{(r+k+1) \alpha, \ldots, n \alpha\} \subseteq\{r+k+1, \ldots, n\}$ will be an idempotent in $\mathcal{O}_{x}$ if and only if the mapping

$$
\left(\begin{array}{ccc}
r+k+1 & \ldots & n \\
(r+k+1) \alpha & \ldots & n \alpha
\end{array}\right)
$$

is an idempotent in $\mathcal{O}_{\{r+k+1, \ldots, n\}}$. Thus

$$
\left|A_{r, r+k}\right|=\phi(n-r-k) \quad(k=0, \ldots, n-r-1) .
$$

Since $\left|A_{r, n}\right|=1$, it now follows from (2.5) that

$$
\phi_{r}(n)=\phi(n-r)+\phi(n-r-1)+\ldots+\phi(1)+1 .
$$

Substituting this in (2.4) we obtain that

$$
\phi(n)=\phi(n-1)+2 \phi(n-2)+3 \phi(n-3)+\ldots+(n-1) \phi(1)+n,
$$

from which it readily follows that, for all $n \geqq 3$,

$$
\phi(n)-3 \phi(n-1)+\phi(n-2)=0 .
$$

If we make the direct observation that $\phi(1)=1, \phi(2)=3$, it now follows by elementary methods (see for example (1)) that

$$
\phi(n)=\frac{1}{2^{n} \sqrt{5}}\left\{(3+\sqrt{5})^{n}-(3-\sqrt{5})^{n}\right\} .
$$


The alternative formula given in the theorem follows from this by the binomial theorem. $\dagger$

Remark. The number of idempotents of defect 1 is found by observing (a) that there are $n-1$ choices of the unique element $u$ for which $u \alpha=(u+1) \alpha$, and (b) that to each such choice of $u$ there correspond two idempotents $\varepsilon, \eta$ (say) where

$$
u \varepsilon=(u+1) \varepsilon=u, \quad u \eta=(u+1) \eta=u+1 .
$$

Thus there are $2 n-2$ idempotents of defect 1 and so, by virtue of the remarks immediately following the statement of Theorem 1.1, the semigroup $\mathcal{O}_{X}$ is generated by $2 n-1$ idempotents, namely the $2 n-2$ idempotents of defect 1 and the single idempotent of defect zero.

\section{Sets of order-type $\omega$}

The most natural case to consider next is where the set $X$ has order-type $\omega$, so that effectively we may identify $X$ with the set $N=\{1,2,3, \ldots\}$ of natural numbers. Here the problem of describing the elements of $\mathcal{O}_{X}$ that are expressible as products of idempotents is vastly more difficult and $I$ have as yet been unable to solve it. A partial result may serve to indicate the complexity of the situation.

If $\alpha \in \mathcal{O}_{X}$, let us call the subsets $x \alpha^{-1}(x \in X \alpha)$ of $X$ the components of $\alpha$; they are the classes of the equivalence relation

$$
\pi_{\alpha}=\{(x, y) \in X \times X: x \alpha=y \alpha\},
$$

and are convex subsets $C$ of $X$, in the sense that whenever $x, y \in C$ with $x<y$ then $z \in C$ for every $z$ such that $x \leqq z \leqq y$. A component will be called trivial if it consists of a single element. We shall use the standard notation $X / \pi_{\alpha}$ for the set of components of $\alpha$.

Let $\mathscr{B}_{X}$ be the subset of $\mathscr{O}_{X}$ consisting of all increasing mappings for which the components are finite and bounded in size. If $\alpha \in \mathscr{B}_{X}$, let us denote

by $c(\alpha)$.

$$
\sup \left\{\left|x \alpha^{-1}\right|: x \in X \alpha\right\}
$$

Lemma 3.1. $\mathscr{B}_{X}$ is a subsemigroup of $\mathcal{O}_{X}$.

Proof. If $\alpha, \beta \in \mathscr{B}_{X}$ then, for every $x$ in $X$,

$$
x \alpha \beta-x=((x \alpha) \beta-x \alpha)+(x \alpha-x) \geqq 0
$$

and so $\alpha \beta$ is increasing. Also, for every $y$ in $X \alpha \beta$,

$$
y(\alpha \beta)^{-1}=\bigcup_{z \in y \beta-1 \cap X \alpha} z \alpha^{-1} \quad \text { (a disjoint union) }
$$

$\dagger$ Dr. D. Monk has pointed out (in a letter to me) that the formula in Theorem 2.3 appears in a paper (7) by Mullin and Stanton as the number of "spanning trees" of a graph consisting of $n+1$ vertices $v_{1}, \ldots, v_{n}, v_{\infty}$, where $v_{l}$ is joined to $v_{t+1}(i=1, \ldots, n-1)$ and each of $v_{1}, \ldots, v_{n}$ is joined to $v_{\infty}$. The connection between this number and the number of idempotents in $O_{x}$ is not hard to establish.

Dr. Monk also points out that the numbers $\phi(n)$ are alternate Fibonacci numbers: $\phi(n)=F_{2 n}$, where $F_{1}=F_{2}=1$ and $F_{n}=F_{n-1}+F_{n-2}(n \geqq 3)$. 
and so

$$
\left|y(\alpha \beta)^{-1}\right|=\sum_{z \in y \beta^{-1} \cap X \alpha}\left|z \alpha^{-1}\right| \leqq c(\alpha) c(\beta) .
$$

Thus $\mathscr{B}_{X}$ is closed under multiplication.

It is possible to give a description of the subsemigroup generated by the idempotents of $\mathscr{B}_{X}$, but some definitions are necessary before the theorem can be stated.

First, let us say that $\alpha$ is narrow if the set $\{x \alpha-x: x \in X\}$ of non-negative integers is bounded. If $C=c \alpha^{-1}=\{x, x+1, \ldots, x+k\}$ is a component of $\alpha$, let us denote $c-(x+k)$ by $\sigma(C)$, and let us call $C$ a shifting component if $\sigma(C)>0$. If $\alpha$ is narrow, then $\left\{\sigma(C): C \in X / \pi_{\alpha}\right\}$ is bounded; let us denote

$$
\sup \left\{\sigma(C): C \in X / \pi_{\alpha}\right\}
$$

by $b(\alpha)$. Conversely, if $\left\{\sigma(C): C \in X / \pi_{\alpha}\right\}$ is bounded, with supremum $b(\alpha)$, then $\alpha$ is narrow, since for every $x$ in $X$ (lying in some component $C$, say),

$$
x \alpha-x \leqq \sigma(C)+|C| \leqq b(\alpha)+c(\alpha) .
$$

Notice that if $\alpha \in \mathscr{B}_{X}$ then $\alpha$ is idempotent if and only if $b(\alpha)=0$.

An element

$$
\alpha=\left(\begin{array}{cccc}
C_{1} & C_{2} & C_{3} & \ldots \\
c_{1} & c_{2} & c_{3} & \ldots
\end{array}\right) \quad\left(c_{1}<c_{2}<c_{3}<\ldots\right)
$$

of $\mathscr{B}_{X}$ will be called neat if there exists a non-negative integer $n$ such that for every shifting component $C_{i}$ of $\alpha$ one or more of the components

$$
C_{i+1}, C_{i+2}, \ldots, C_{i+n+1}
$$

is non-trivial. The smallest $n$ with this property will be denoted by $n(\alpha)$. If there are no shifting components (which is the case if and only if $\alpha$ is idempotent), we define $n(\alpha)=0$.

We note that an element $\alpha$ of $\mathscr{B}_{X}$ of finite shift is both narrow and neat. The former is obvious; to see the latter, observe that $\alpha$ must either be idempotent or must have a last shifting component $C_{i}=\{\ldots, x\}$. Since $x \alpha \geqq x+1$ and since $(x+1) \alpha>x \alpha$, it follows that $(x+1) \alpha \geqq x+2$. The component $C_{i+1}$ by assumption does not shift and so must be non-trivial. It now follows that every shifting component of $\alpha$ is a bounded distance below a non-trivial component.

Remark. If $\alpha\left(\in \mathscr{B}_{X}\right)$ is both narrow and neat and has infinite shift, then $\alpha$ has infinitely many non-trivial components, for otherwise all components from a certain point on would have to be trivial, and infinitely many of them (in fact all of them) would be shifting components. This would contradict the assumption of neatness. Thus, in the terminology of (5), $\alpha$ has infinite collapse.

It is also the case that $\alpha$ has infinite defect. If not, then from some point on there are no gaps in $X \alpha$ : more precisely, there exists $n$ such that

$$
\{x \alpha: x \geqq n\}=\{y: y \geqq n \alpha\} \text {. }
$$


If $n \alpha-n=k(\geqq 0)$ then by virtue of the lack of gaps in $X \alpha$ we have that $0 \leqq x \alpha-x \leqq k$ for every $x \geqq n$. If $k=0$ we have an immediate contradiction to the assumption that $\alpha$ has infinite shift. If $k \geqq 1$ then, since $\alpha$ is neat, there exists a non-trivial component $C=\{m, m+1, \ldots\}$ of $\alpha$ whose members are greater than $n$. Since $m \alpha-m \leqq k$ it follows that

$$
(m+1) \alpha-(m+1)=m \alpha-(m+1) \leqq k-1 ;
$$

hence $y \alpha-y \leqq k-1$ for every $y \geqq m+1$. We have seen that there are infinitely many non-trivial components of $\alpha$, and each one of them effects a decrease of at least one in the value of $x \alpha-x$ beyond that point. Eventually we find a natural number $p$ such that $z \alpha-z=0$ for all $z \geqq p$, which contradicts the hypothesis that $\alpha$ has infinite shift.

To summarize, we have observed that elements of infinite shift that are both neat and narrow have infinite defect and collapse, and so, by Theorem III of (5), are expressible as products of idempotents, provided we take no account of the ordering of $X$. We shall see shortly that it is possible to express such elements as products of order-preserving idempotents.

Some examples may serve to clarify the concepts introduced above. If

$$
(2 m-1) \alpha=2 m \alpha=2^{m} \quad(m=1,2, \ldots)
$$

then $\alpha$ is neat but not narrow. If

$$
m \beta= \begin{cases}m+2 & \text { for } m=1,4,9,16, \ldots \\ m+1 & \text { otherwise, }\end{cases}
$$

then $\beta$ has infinite shift, defect and collapse and is narrow, but is not neat. If

$$
m \gamma= \begin{cases}m+2 & \text { if } m \equiv 3 \quad(\bmod 4) \\ m+1 & \text { otherwise }\end{cases}
$$

then $\gamma$ is both narrow and neat, with

$$
b(\gamma)=1, \quad n(\gamma)=2 .
$$

Theorem 3.2. An element of the semigroup $\mathscr{B}_{X}$ is expressible as a product of idempotents in $\mathscr{B}_{X}$ if and only if it is both narrow and neat.

Proof. We show first that the subset $\mathscr{N}_{X}$ of $\mathscr{B}_{X}$ consisting of those elements that are both narrow and neat is a subsemigroup. Certainly, if $\alpha$ and $\beta$ are narrow then

$$
x \alpha \beta-x=((x \alpha) \beta-x \alpha)+(x \alpha-x)
$$

and so $\{x \alpha \beta-x: x \in X\}$ is bounded.

Suppose now that

$$
\alpha=\left(\begin{array}{ccc}
C_{1} & C_{2} & \ldots \\
c_{1} & c_{2} & \ldots
\end{array}\right), \quad \beta=\left(\begin{array}{ccc}
D_{1} & D_{2} & \ldots \\
d_{1} & d_{2} & \ldots
\end{array}\right)
$$

are both neat. Writing $\alpha \beta$ as

$$
\left(\begin{array}{ccc}
E_{1} & E_{2} & \cdots \\
e_{1} & e_{2} & \cdots
\end{array}\right)
$$


let us consider a shifting component $E_{j}$ of $\alpha \beta$. Since $\pi_{\alpha} \subseteq \pi_{\alpha \beta}$, the component $E_{j}$ is a union of components of $\alpha$. It must moreover be a "convex" union in the sense that if $C_{p} \subset E_{j}, C_{q} \subset E_{j}$ with $p<q$, then $C_{r} \subset E_{j}$ for all $r$ such that $p \leqq r \leqq q$. The following lemma is relevant.

Lemma 3.3. Let $C_{i}, E_{j}$ be shifting components of $\alpha, \alpha \beta$ respectively, such that $C_{i} \subseteq E_{j}, C_{i+1} \notin E_{j}$. If, for some $n \geqq 0$, the component $C_{i+n+1}$ of $\alpha$ is nontrivial, then at least one of the components $E_{j+1}, \ldots, E_{j+n+1}$ of $\alpha \beta$ is non-trivial.

Proof. The component $C_{i+n+1}$ is contained in some (necessarily non-trivial) component $E_{r}$ of $\alpha \beta$. Since

$$
E_{r} \alpha \beta=\left(C_{i+n+1} \alpha\right) \beta \geqq\left(C_{i+1} \alpha\right) \beta>\left(C_{i} \alpha\right) \beta=E_{j} \alpha \beta,
$$

we must have $r>j$. Also, since components of $\alpha \beta$ are convex unions of components of $\alpha$, we must have $r \leqq j+n+1$. Thus the lemma is proved.

We have chosen a shifting component $E_{j}$ of $\alpha \beta$. Let $C_{i}$ be the component of $\alpha$ with the property that $C_{i} \subseteq E_{j}$ and $C_{i+1} \ddagger E_{j}$, and let us denote the largest element of $C_{t}$ by $x$. Certainly $x \alpha \beta>x$. If $x \alpha>x$ then $C_{i}$ is a shifting component and the neatness of $\alpha$ allows us to assume that $C_{i+n+1}$ is non-trivial for some $n \leqq n(\alpha)$. Lemma 3.3 now implies that there is a non-trivial component $E_{j+k+1}$ of $\alpha \beta$, with

$$
0 \leqq k \leqq n \leqq n(\alpha) .
$$

It is, however, also possible to have $x \alpha=x$ (with $x \beta>x$ ), and this case proves much more troublesome. The element $x$ lies in some component $D_{p}$ of $\beta$, and it is convenient to separate two cases:

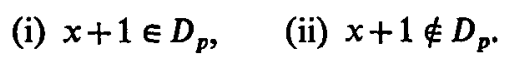

In case (i) we must have $(x+1) \alpha>x+1$, since $(x+1) \alpha=x+1$ would imply that

$$
(x+1) \alpha \beta=(x+1) \beta=x \beta=x \alpha \beta
$$

and so $x+1 \in E_{j}$ contrary to hypothesis. Thus the component $C_{i+1}$ (which contains $x+1$ ) is either non-trivial or is a shifting component of $\alpha$. If it is nontrivial then it is contained in $E_{j+1}$, a necessarily non-trivial component of $\alpha \beta$. If $C_{i+1}$ is trivial, then either $E_{j+1}$ is non-trivial, or $C_{i+1}=E_{j+1}$ and so $C_{i+2} \nsubseteq E_{j+1}$. In the latter case Lemma 3.3 applies and the general conclusion for case (i) is that there is a non-trivial component $E_{j+k+1}$, where $k \geqq 0$ and where at worst

$$
k \leqq n(\alpha)+1 \text {. }
$$

In case (ii) $x$ is the greatest element of $D_{p}$ and so $D_{p}$ is a shifting component; hence by neatness of $\beta$ there is a non-trivial component

$$
D_{p+m+1}=\{y, y+1, \ldots\}
$$

of $\beta$ with $m \leqq n(\beta)$. Let us in fact assume that we have chosen $m$ as small as 
possible, so that the components $D_{p+1}, \ldots, D_{p+m}$ are all trivial. It follows that

$$
y-x=\left|D_{p+1}\right|+\ldots+\left|D_{p+m}\right|+1=m+1 .
$$

If there is no shifting or non-trivial component of $\alpha$ between $x$ and $y$, then

$$
C_{i}=\{\ldots, x\}, \quad C_{i+1}=\{x+1\}, \ldots, \quad C_{i+y-x}=C_{i+m+1}=\{y\},
$$

where $x \alpha=x,(x+1) \alpha=x+1, \ldots, y \alpha=y$. If $(y+1) \alpha=y+1$ then

$$
y \alpha \beta=(y+1) \alpha \beta
$$

and so $\{y, y+1\}$ is all or part of a non-trivial component of $\alpha \beta$. Otherwise $(y+1) \alpha>y+1$ and so the component $C_{i+m+2}$ of $\alpha$ is either non-trivial or shifting (or both). If it is non-trivial then it is part of a non-trivial component of $\alpha \beta$. The very worst that can happen is that $C_{i+m+2}$ and all the components

$$
E_{j+1}, \ldots, E_{j+m+2} \quad\left(=C_{i+m+2}\right)
$$

are trivial. But in this case the lemma applies, and there must be a non-trivial component $E_{j+k+1}$, where

$$
k \leqq m+2+n(\alpha) \leqq ! n(\alpha)+n(\beta)+2 .
$$

The general conclusion is that $\alpha \beta$ is neat and that

$$
n(\alpha \beta) \leqq n(\alpha)+n(\beta)+2 .
$$
if

Remark. The upper bound $n(\alpha)+n(\beta)+2$ can be attained. For example,

$$
\alpha=\left(\begin{array}{lllllllll}
1 & 2 & 3 & 4 & 5 & 6 & 7 & 8 & \ldots \\
1 & 2 & 3 & 5 & 6 & 7 & 7 & 8 & \ldots
\end{array}\right), \quad \beta=\left(\begin{array}{lllllllll}
1 & 2 & 3 & 4 & 5 & 6 & 7 & 8 & \ldots \\
2 & 3 & 4 & 4 & 5 & 6 & 7 & 8 & \ldots
\end{array}\right)
$$

are elements of finite shift (in which $x \alpha=x \beta=x$ for all $x>8$ ), then

$$
\alpha \beta=\left(\begin{array}{lllllllll}
1 & 2 & 3 & 4 & 5 & 6 & 7 & 8 & \ldots \\
2 & 3 & 4 & 5 & 6 & 7 & 7 & 8 & \ldots
\end{array}\right),
$$

and it is easy to see that $n(\alpha)=n(\beta)=1$, while $n(\alpha \beta)=4$.

To complete the proof of Theorem 3.2 we must show that every element which is both narrow and neat is expressible as a product of idempotents. As a preliminary, let us define two mappings, $f, g$ from $\mathscr{B}_{X}$ into itself. If

is in $\mathscr{B}_{X}$ we define $\alpha f: X \rightarrow X$ by

$$
\alpha=\left(\begin{array}{llll}
C_{1} & C_{2} & C_{3} & \ldots \\
c_{1} & c_{2} & c_{3} & \cdots
\end{array}\right)
$$

$$
x(\alpha f)=x_{i} \text { for every } x \text { in } C_{i} \quad(i=1,2, \ldots),
$$

where $x_{i}$ is the greatest element of $C_{\mathrm{i}}$. Notice that $\alpha f$ is an idempotent in $\mathscr{B}_{\mathrm{X}}$, and that $\alpha f=\alpha$ if and only if $\alpha$ is idempotent. 
Next, we define $\alpha g: X \rightarrow X$ by

$$
\begin{aligned}
& x_{i}(\alpha g)=c_{i} \quad(i=1,2, \ldots), \\
& y(\alpha g)=c_{1} \quad \text { if } y \in C_{1} \backslash\left\{x_{1}\right\}, \\
& y(\alpha g)=c_{i-1} \text { if } y \in C_{i} \mid\left\{x_{i}\right\} \text { and } y \leqq c_{i-1}, \\
& y(\alpha g)=c_{i} \quad \text { if } y \in C_{i} \backslash\left\{x_{i}\right\} \text { and } y>c_{i-1} .
\end{aligned}
$$

It is easy to see that $\alpha g \in \mathscr{B}_{X}$ and that

$$
(\alpha f)(\alpha g)=\alpha .
$$

For example, if

is defined by

$$
\gamma=\left(\begin{array}{lllllllll}
1 & 2 & 3 & 4 & 5 & 6 & 7 & 8 & \ldots \\
4 & 4 & 4 & 5 & 8 & 8 & 8 & 9 & \ldots
\end{array}\right)
$$

$$
\begin{gathered}
x y=x+3 \text { if } x \equiv 1 \quad(\bmod 4) \\
x+2 \text { if } x \equiv 2 \quad(\bmod 4) \\
x+1 \text { otherwise, }
\end{gathered}
$$

then

$$
\left.\gamma f=\left(\begin{array}{lllllllll}
1 & 2 & 3 & 4 & 5 & 6 & 7 & 8 & \ldots \\
3 & 3 & 3 & 4 & 7 & 7 & 7 & 8 & \ldots
\end{array}\right), \quad \gamma g=\left(\begin{array}{llllllllll}
1 & 2 & 3 & 4 & 5 & 6 & 7 & 8 & 9 & \ldots \\
4 & 4 & 4 & 5 & 5 & 8 & 8 & 9 & 9 & \ldots
\end{array}\right) .\right]
$$

If $\alpha$ is idempotent then $c_{i}=x_{i}(i=1,2, \ldots)$ and so $y \in C_{i}(i>1)$ implies that $y>x_{i-1}=c_{i-1}$. Thus $y(\alpha g)=c_{i}$ for every $y$ in $C_{i}$ and for every $i$ (including $i=1$ ), and so $\alpha g=\alpha$. Conversely, if $\alpha g=\alpha$ then for each $i \geqq 2$ we must have $y>c_{i-1}$ for every $y$ in $C_{i}$. In particular,

$$
x_{i-1}+1>c_{i-1}=x_{i-1} \alpha \geqq x_{i-1},
$$

from which it follows that $c_{i-1}=x_{i-1}$. Thus $\alpha$ is idempotent.

In summary, we have shown that $\alpha$ is idempotent if and only if $\alpha f=\alpha g=\alpha$.

For a given element $\alpha$ in $\mathscr{B}_{X}$ we now define two sequences

$$
\alpha_{0}, \alpha_{1}, \alpha_{2}, \ldots \text { and } \varepsilon_{1}, \varepsilon_{2}, \varepsilon_{3}, \ldots
$$

by setting $\alpha_{0}=\alpha$ and

$$
\varepsilon_{r}=\alpha_{r-1} f, \quad \alpha_{r}=\alpha_{r-1} g \quad(r=1,2, \ldots) .
$$

By virtue of (3.4) we have

and so

$$
\alpha_{r-1}=\varepsilon_{r} \alpha_{r}
$$

$$
\alpha=\varepsilon_{1} \varepsilon_{2} \ldots \varepsilon_{r} \alpha_{r} \quad(r=1,2, \ldots),
$$

where $\varepsilon_{1}, \ldots, \varepsilon_{r}$ are idempotents. We shall show that if $\alpha$ is narrow and neat then $\alpha_{r}$ is idempotent for some sufficiently large $r$.

Since

$$
X \alpha=X \alpha_{1}=X \alpha_{2}=\ldots=\left\{c_{1}, c_{2}, \ldots\right\}
$$


we can, for each $k \geqq 1$, establish a natural one-one correspondence between the set of components of $\alpha_{k}$ and that of $\alpha$, whereby the component $C_{i}^{(k)}=c_{i} \alpha_{k}^{-1}$ of $\alpha_{k}$ corresponds to the component $C_{i}=c_{i} \alpha^{-1}$ of $\alpha$.

Since (for each $i \geqq 1$ ) the component $C_{i}^{(1)}=c_{i} \alpha_{1}^{-1}$ contains $x_{i}$ and may contain some elements of $C_{i+1}$, we certainly have that

$$
\sigma\left(C_{i}^{(1)}\right) \leqq \sigma\left(C_{i}\right),
$$

where (as before) $\sigma\left(C_{i}\right)$ is the difference between the image of $C_{i}$ and the largest member of $C_{i}$. In particular,

$$
\sigma\left(C_{i}^{(1)}\right)=0 \text { if } \sigma\left(C_{i}\right)=0 .
$$

If we make a further assumption we can obtain a strict inequality in (3.6). Precisely, if $\sigma\left(C_{i}\right)>0$ and if $C_{i+1}$ is non-trivial, then

To see this, observe that

$$
\sigma\left(C_{i}^{(1)}\right)<\sigma\left(C_{i}\right) .
$$

$$
C_{i}=\left\{\ldots, x_{i}\right\}, \quad C_{i+1}=\left\{x_{i}+1, x_{l}+2, \ldots\right\} .
$$

Now $c_{i}>x_{i}$ by assumption and so $x_{i}+1 \leqq c_{i}$. It follows by the definition of $\alpha_{1}$ that $\left(x_{i}+1\right) \alpha=c_{i}$ and so

$$
C_{i}^{(1)}=\left\{\ldots, x_{i}, x_{i}+1, \ldots\right\} .
$$

The inequality (3.7) follows, since certainly

$$
\sigma\left(C_{i}^{(1)}\right) \leqq c_{i}-\left(x_{i}+1\right)<c_{i}-x_{i}=\sigma\left(C_{i}\right) .
$$

[As an example of this, consider the component $C_{2}=\{4\}$ of the $\gamma$ in (3.5), where $C_{2}^{(1)}=\{4,5\}, \sigma\left(C_{2}\right)=1, \sigma\left(C_{2}^{(1)}\right)=0$.]

A repetition of the same argument establishes the following lemma, in which $C_{i}^{(0)}, C_{i+1}^{(0)}$ are to be interpreted as $C_{i}, C_{i+1}$ respectively.

Lemma 3.8. For every $k \geqq 0$,

$$
\sigma\left(C_{i}^{(k+1)}\right) \leqq \sigma\left(C_{i}^{(k)}\right) .
$$

The inequality is strict provided $\sigma\left(C_{i}^{(k)}\right)>0$ and $C_{i+1}^{(k)}$ is non-trivial.

As a consequence of this lemma, we have that if $\alpha$ is narrow then so are $\alpha_{1}, \alpha_{2}, \ldots$, and

$$
b(\alpha) \geqq b\left(\alpha_{1}\right) \geqq b\left(\alpha_{2}\right) \geqq \ldots .
$$

If $\sigma\left(C_{i}\right)>0$ and $C_{i+1}$ is trivial, then if $\alpha$ is neat there is a non-trivial component $C_{i+n+1}$ where $1 \leqq n \leqq n(\alpha)$. Here we have

$$
\begin{gathered}
C_{i}=\left\{\ldots, x_{i}\right\}, \quad C_{i+1}=\left\{x_{i}+1\right\}, \ldots, \quad C_{i+n}=\left\{x_{i}+n\right\}, \\
C_{i+n+1}=\left\{x_{i}+n+1, x_{i}+n+2, \ldots\right\} .
\end{gathered}
$$

In fact all the components $C_{i+1}, \ldots, C_{i+n}$ must shift; for $c_{i} \geqq x_{i}+1$ by assumption and so

$$
c_{i+1} \geqq c_{i}+1 \geqq x_{i}+2 ;
$$


thus $C_{i+1}$ shifts, and the same argument can be applied repeatedly to show that $C_{i+2}, \ldots, C_{i+n}$ all shift. Thus $c_{i+n} \geqq x_{i}+n+1$ and so $\left(x_{i}+n+1\right) \alpha_{1}=c_{i+n}$. The component $C_{i+n}^{(1)}$ of $\alpha_{1}$ thus contains at least one element besides $x_{i}+n$ and so is non-trivial.

Applying this argument in general, we obtain the following lemma, which holds for every $k \geqq 0$.

Lemma 3.10. If $\sigma\left(C_{i}^{(k)}\right)>0$, if $C_{i+1}^{(k)}, \ldots, C_{i+n}^{(k)}$ are trivial, and if $C_{i+n+1}^{(k)}$ is non-trivial $(n \geqq 1)$, then $\sigma\left(C_{i}^{(k+1)}\right)=\sigma\left(C_{i}^{(k)}\right)$ and $C_{i+n}^{(k+1)}$ is non-trivial.

A final lemma is

Lemma 3.11. If $\alpha$ is neat, then every $\alpha_{k}$ is neat, and

$$
n(\alpha) \geqq n\left(\alpha_{1}\right) \geqq n\left(\alpha_{2}\right) \geqq \cdots
$$

Proof. It is enough to show that $n(\alpha) \geqq n\left(\alpha_{1}\right)$. To show that $\alpha$ is neat we consider an arbitrary shifting component $C_{i}^{(1)}$ of $\alpha_{1}$. Certainly $C_{i}$ is a shifting component of $\alpha$, by (3.6). If $C_{i+1}$ is trivial, Lemma 3.10 implies that $C_{i+n}^{(1)}$ is a non-trivial component of $\alpha_{1}$, with $1 \leqq n \leqq n(\alpha)$. If $C_{i+1}$ is non-trivial, however, the lemma gives no useful information, and it is possible that $C_{i+1}^{(1)}$ is trivial.

In this case, since $C_{l}^{(1)}$ shifts it follows that $C_{i+1}^{(1)}$ shifts; hence $C_{i+1}$ shifts by virtue of (3.6). We are supposing that $\alpha$ is neat, and so for some $n$ such that $0 \leqq n \leqq n(\alpha)$ the component $C_{i+n+2}$ is non-trivial. If $n \geqq 1$ Lemma 3.10 implies that $C_{i+n+1}^{(1)}$ is non-trivial. The case $n=0$ cannot in fact arise, since if $C_{i+2}$ were non-trivial then $C_{i+1}^{(1)}$ would, by the argument used to establish (3.7), be non-trivial, and we are assuming that this is not the case.

To summarize, we have shown that for all shifting components $C_{i}^{(1)}$ of $\alpha_{1}$, at least one of the components

$$
C_{i+1}^{(1)}, \ldots, C_{i+n(\alpha)+1}^{(1)}
$$

is non-trivial. Hence $\alpha_{1}$ is neat and $n\left(\alpha_{1}\right) \leqq n(\alpha)$.

It is now a consequence of Lemmas 3.8 and 3.10 that if $k \geqq n(\alpha)+1$ then

$$
\sigma\left(C_{i}^{(k)}\right) \leqq \sigma\left(C_{i}\right)-1
$$

for all components $C_{i}$ such that $\sigma\left(C_{i}\right)>0$. But $n\left(\alpha_{k}\right) \leqq n(\alpha)$ and so if

$$
k \geqq 2(n(\alpha)+1)
$$

we obtain by a repetition of the same argument that

$$
\sigma\left(C_{i}^{(k)}\right) \leqq \sigma\left(C_{i}\right)-2
$$

for all components $C_{i}$ such that $\sigma\left(C_{i}\right)>1$. Eventually we find that, for all $k \geqq b(\alpha)(n(\alpha)+1)$,

$$
\sigma\left(C_{i}^{(k)}\right)=0 \text { for } i=1,2, \ldots
$$

and so $\alpha_{k}$ is idempotent.

This completes the proof of Theorem 3.2. 


\section{REFERENCES}

(1) Clement V. Durell and A. Robson, Advanced Algebra, vol. II (London, 1937).

(2) J. A. ERDos, On products of idempotent matrices, Glasgow Math. J. 8 (1967), 118-122.

(3) Bernard HaRris, A note on the number of idempotent elements in symmetric semigroups, Amer. Math. Monthly 74 (1967), 1234-1235.

(4) BERNARD HaRris and Lowell Schoenfeld, The number of idempotent elements in symmetric semigroups, $J$. Combinatorial Theory 3 (1967), 122-135.

(5) J. M. HowIE, The subsemigroup generated by the idempotents of a full transformation semigroup, J. London Math. Soc. 41 (1966), 707-716.

(6) K. D. MaGmL, Jr., Semigroups of functions generated by idempotents, $J$. London Math. Soc. 44 (1969), 236-242.

(7) R. C. Mullin and R. G. Stanton, A combinatorial property of spanning forests in connected graphs, J. Combinatorial Theory 3 (1967), 236-243.

(8) M. TAINITER, A characterization of idempotents in semigroups, J. Combinatorial Theory 5 (1968), 370-373.

UNIVERSITY OF ST. ANDREWS 